\title{
Illusione del dialetto e ambivalenza semantica nei Malavoglia
}

Secondo Riccardo Ambrosini, con critica linguistica si intende, da un punto di vista operativo, l'esame dei rapporti tra l'opera letteraria, con quella data forma espressiva scelta dall'autore, e le intenzioni espressive che l'hanno prodotta, in quanto, da un punto di vista teorico, il rapporto tra forma espressiva e intenzione espressiva è di natura dialettica (8). Nulla di più vero quando si parla della narrativa verghiana e delle scelte linguistiche dello scrittore siciliano in rapporto al nuovo credo veristico dell'Italia post-unitaria che si propone di studiare oggettivamente la società in tutti i suoi aspetti con i metodi delle scienze naturali e sociologiche. Un'analisi linguistica dell'opera letteraria verghiana si prefigge quindi di evidenziare gli elementi dialettici nei quali essa è inserita e le tensioni interne tra forma espressiva e intenzione espressiva. In altre parole, Verga è veramente riuscito ad applicare nei Malavoglia i principi veristici di mimesis del linguaggio popolare?

In questo contesto molto è stato detto e scritto a proposito della lingua utilizzata dal Verga nei Malavoglia, sulla maniera in cui l'autore trapianta parole, sintagmi e costrutti sintattici siciliani nella forma italiana corrispondente. Il costante rifiuto dello scrittore di scrivere I Malavoglia in dialetto siciliano corrisponde alla sua ferma determinazione di creare un'opera che rispecchi sì la realtà popolare quotidiana dell'umile gente siciliana, ma che sia al tempo stesso accessibile alla maggioranza del pubblico italiano. Come osserva giustamente Vitilio Masiello, si tratta di

un pastiche linguistico, a metà strada tra lingua e dialetto (in funzione di un illusorio progetto di acquisizione alla cultura borghese di una realtà umana e culturale inedita e diversa), vitalizzato da una forte capacità connotativa e caratteriologica in virtù della sua idoneità a dar forma a comportamenti, sensazioni, sentimenti e pensieri di una umanità primitiva ed elementare. (97)

Critici eminenti come Ettore Caccia, Giovanni Cecchetti, Luigi Russo, Giovanni Nencioni, Giacomo Devoto, Leo Spitzer, Enrico Giachery, Giacomo Debenedetti, Gino Raya, Natalino Sapegno, Riccardo Ambrosini, Vitilio Masiello, Gabriella Alfieri e altri, hanno esaminato dettagliatamente le varie trasposizioni morfologiche, sintattiche e lessicali operate dal Verga dal dialetto alla lingua italiana, e hanno riconosciuto l'eccellente capacità dello scrittore di rendere l'ambiente di Trezza creando l'illusione della parlata dialettalepopolare. Tuttavia, al tempo stesso, molti di loro hanno dovuto ammettere che si tratta di un dialetto illustre, trasfigurato, mitificato, ben lontano dalla 
genuina parlata popolare siciliana, in definitiva, di una lingua letteraria che non è esente dalla forma convenzionale trionfante nella letteratura italiana dal Petrarca al Manzoni. ${ }^{1}$ Infatti l'illusione del dialetto, creata per rappresentare realisticamente l'ambiente popolare, si sfalda in quelle parafrasi dal siciliano che non reggono nella traduzione italiana, dando luogo ad ambivalenze semantiche incapaci di riflettere l'originario nucleo dialettale-popolare.

La mia analisi si riallaccia agli studi precedenti condotti sull'originale linguaggio verghiano, sui vari trapianti dialettali nel testo narrativo, e in particolare all'ampia analisi condotta da Gabriella Alfieri sulle traduzioni dei proverbi nei Malavoglia. Servendomi dello studio dell'Alfieri come punto di partenza, è mia intenzione approfondire e sviluppare la problematica della traduzione nel romanzo verghiano, usando la metodologia derridiana che individua la dispersione e il deferimento dei significanti nel testo letterario. Lo scopo di questo studio è di mostrare come ogni finalità di organizzazione e totalizzazione nella lingua letteraria venga continuamente problematizzata, come la finzione narrativa riveli la sua natura illusoria e artificiale, come in definitiva la lingua sia contraddistinta da una problematicità inerente alla sua funzione operativa, problematicità che offusca tanto il processo di significazione quanto il tentativo di trasposizione da una lingua all'altra.

\section{Verga e il tentativo dell'impersonalità: l'illusione del dialetto}

Nell'introduzione all'“Amante di Gramigna' la dedica del Verga al Farina annuncia il nuovo credo veristico:

Eccoti non un racconto, ma l'abbozzo di un racconto. Esso almeno avrà il merito di esser brevissimo, e di essere storico-un documento umano, come dicono oggi. ... Io te lo ripeto così come l'ho raccolto pei viottoli dei campi, press'a poco con le medesime parole semplici e pittoresche della narrazione popolare, e tu veramente preferirai di trovarti faccia a faccia col fatto nudo e schietto, senza stare a cercarlo fra le linee del libro, attraverso la lente dello scrittore. ${ }^{2}$

La principale vocazione del Verga è il linguaggio popolare, nel quale egli scopre immediatezza ed efficacia di contro all'artificiosa tradizione letteraria dalla quale vuole liberarsi. Lo scrittore "cerca così di accostarsi alla materia degli umili e casalinghi affetti, con l'atteggiamento dell'uomo di mondo che si è distolto con ripugnanza da un'esistenza sterile e viziata" (Sapegno 161). Lo scopo maggiore del verismo letterario, in opposizione alle languide fantasie romantiche, è di rappresentare individui reali nell'ambiente sociale contemporaneo, attenendosi quanto più possibile all'osservazione oggettiva e alla documentazione scientifica. Questi principi il verismo italiano li ha ereditati dal naturalismo francese, secondo il quale i fatti reali devono essere presentati con distacco da parte dello scrittore che deve rimanere invisibile dietro la sua creazione. Il 10 luglio 1879, Francesco De Sanctis proclamava: "il motto di un'arte seria è questo: poco parlare di noi, e far parlare molto le cose" (Cecchetti 182). ${ }^{3}$ 
L'impersonalità allora è una conseguenza del linguaggio popolare, il quale tanto più brillerà quanto più sarà colto alle sue fonti parlate, "senza la lente dello scrittore". Ettore Caccia riassume i canoni del verismo e il principio dell'impersonalità come un felice tentativo del Verga di ribellarsi contro l'accademismo retorico (231). Tuttavia il principio, come tutte le enunciazioni astratte, presenta difficoltà di applicazione. Infatti, è veramente possibile costruire un'opera d'arte che "sembrerà essersi fatta da sé, senza serbare alcun punto di contatto col suo autore"? E se Verga vuole un linguaggio popolare perché non riproduce direttamente il dialetto, invece di servirsi di una traduzione che si rivela spesso ambigua e forzata? Ma cerchiamo di seguire più da vicino il programma letterario del Verga per scoprime le tensioni e contraddizioni interne. Il 19 luglio 1880 lo scrittore siciliano scrive al Treves: "l'importanza del realismo sta in ciò che più si riesce a rendere immediata l'impressione artistica, meglio questa sarà oggettiva, quindi vera o reale come volete, ma bella sempre. Ci riuscirò nei Malavoglia?" E il 9 agosto dello stesso anno: "Pel titolo resta adottato I Malavoglia, invece di Padron 'Ntoni .... Mi pare di essere riuscito a dare il rilievo dovuto ai personaggi, metterli nell'ambiente vero e aver reso realmente questo ambiente" (Russo 350).

Per riprodurre realisticamente le chiacchierate della gente del paese il Verga si trova a fronteggiare il problema di quale lingua adoperare, dal momento che i veristi italiani, contrariamente ai naturalisti francesi, hanno intorno a sé una società non ancora omogenea e una lingua nazionale solo a un livello astrattamente letterario. Come osserva giustamente Giovanni Nencioni, in Italia a quel tempo si ha da un lato il purismo, legato a una tradizione di estetismo classico; dall'altro il realismo intriso d'istanze sociologiche, che si riallaccia all'esempio manzoniano. Il problema del Verga è di trovare una lingua adatta a rappresentare il mondo semplice e grezzo dei pescatori siciliani. ${ }^{4}$ Rifiutandosi di usare direttamente il dialetto siciliano, non accessibile alla maggioranza del pubblico italiano, lo scrittore crede di aver trovato la soluzione in una lingua di cui il siciliano costituisce la forma interna, intessuta di espressioni e costrutti sintattici dialettali resi nella forma italiana corrispondente. Verga, in definitiva, vuole attenersi alla sicilianità nelle grandi linee, rendendo il colore locale in un italiano intelligibile a tutti. Egli giustifica il suo rifiuto di usare direttamente il dialetto siciliano in una lettera al Capuana: "E poi con qual costrutto? Per impicciolirci e dividerci da noi stessi? Per diminuirci in conclusione? Vedi se il Porta, che è il Porta, vale il Parini fuori di Milano. Il colore e il sapore locale sì, come hai fatto tu da maestru e io da sculareddu; ma pel resto i polmoni larghi" (Lettere 215).

La lingua dei Malavoglia è quindi un dialetto trasfigurato che, come sottolinea il Devoto, "non raggiunge una classicità dialettale, né realizza un procedimento di evasione, ma innesta per la prima volta una tradizione locale, non mutilata, in una tradizione letteraria, consolidata dall'opera di una scrittore combattente e costruttivo" (Profilo 155). Se rileggiamo il testo dei Malavoglia, rileviamo lo sforzo costante del Verga di mantenere l'illusione 
del dialetto, ricalcando la sintassi siciliana con il frequente uso del che illogico che congiunge due proposizioni senza un'esplicita funzione relativa, con l'uso abbondante di coordinate al posto di subordinate, o ancora con l'immediata trasposizione di sintagmi, parole o proverbi dialettali. ${ }^{5}$

Sarà utile a questo punto riassumere brevemente le osservazioni dei critici a proposito della mirabile capacita dello scrittore siciliano di creare un'impressione del dialetto, prima di discutere i limiti di questa tecnica e le ambiguità interpretative da essa provocate. Innanzitutto, uno dei modi attraverso i quali il Verga riesce a dare l'impressione della parlata popolare è l'uso costante del discorso indiretto libero, o Erlebte Rede, in cui i pensieri e i discorsi dei personaggi vengono inseriti nel racconto senza un'affermazione del tipo "disse che", rendendo impressionisticamente quello che poteva dire il narratore e dando di conseguenza l'illusione del parlato. Per uno studio ampio e dettagliato del discorso indiretto libero rimandiamo all'eccellente saggio di Leo Spitzer, il quale dimostra come l'originalità della tecnica del Verga nei Malavoglia consista non nell'uso dell'Erlebte Rede che appartiene a una tradizione classica, ma "riella filtrazione sistematica della sua narrazione di un romanzo intero, dal primo fino all'ultimo capitolo, attraverso un coro di parlanti popolari semireale che si aggiunge alla narrazione a mezzo di discorsi e gesti (il racconto dialogato). In questo modo, il romanziere aderisce fedelmente a quello che fanno e dicono i suoi personaggi" (305). ${ }^{6}$

Un'interessante analisi condotta da Enrico Giachery rileva nel testo verghiano una serie di periodi lunghi e disarticolati, che intendono parafrasare il periodare siciliano, in cui proposizioni e immagini sono legate mediante un'illimitata presenza di frasi coordinate introdotte dalla congiunzione "e", che sostituiscono gli appropriati rapporti subordinati, restando al di fuori di ogni dipendenza logica (32). ${ }^{7}$ Esempi di questo genere sono numerosissimi nei Malavoglia:

Allora don Franco diceva, fregandosi le mani, che pareva un piccolo Parlamento, e andava a piantarsi dietro il banco, pettinandosi con le dita la barbona, con certo sorriso furbo che pareva si volesse mangiare qualcuno a colazione, e alle volte si lasciava scappare sottovoce delle mezze parole dinanzi alla gente, rizzandosi sulle gambette, e si vedeva che la sapeva più lunga degli altri, tanto che don Giammaria non poteva patirlo e ci si mangiava il fegato, e gli sputava in faccia parole latine. (66)

I gatti grigi sono i migliori, per acchiappare i topi, e andrebbero a scovarli in una cruna d'ago. Ai gatti non conveniva aprire l'uscio di notte, perché una vecchia di Aci Sant'Antonio l'avevano ammazzata così, che i ladri le avevano rubato il gatto tre giomi avanti, e poi glielo avevano riportato mezzo morto di fame a miagolare dietro l'uscio; e la povera donna non sentendosi il cuore di lasciar la bestiola sulla strada a quell'ora, aveva aperto l'uscio, e così s'eran ficcati i ladri in casa. (68)

Numerosi critici, tra cui Ettore Caccia, hanno identificato il frequente uso del che pleonastico, illogico, traducente il ca siciliano, il quale indica l'espressione di uno stato d'animo particolare del personaggio che parla. ${ }^{8}$ Il che è 
inserito nel testo narrativo a legare due proposizioni che non presentano necessariamente un esplicito valore relativo:

Un po' di soldato gli farà bene a quel ragazzo, che il suo paio di braccia gli piaceva meglio di portarsele a spasso la domenica, anziché servirsene per buscarsi il pane. (153)

"Sì, sono qua, comare Mena; sto qua a mangiarmi la minestra, perché quando vi vedo tutti a tavola, col lume, mi pare di non esser tanto solo, che va via anche l'appetito". (76)

Persino mastro Cirino non si faceva più vivo, e lasciava stare di suonare mezzogiomo e l'avemaria, che mangiava il pane del comune anche lui. (211)

Ma [la Longa] non sapeva che doveva partire anche lei quando meno se lo aspettava, per un viaggio nel quale si riposa sempre, sotto il marmo liscio della chiesa; e doveva lasciarli tutti quelli cui voleva bene, e gli erano attaccati al cuore, che glielo strappavano a pezzetti, ora l'uno ora l'altro. (154)

Un'altra caratteristica del dialetto siciliano che Verga trapianta nella sua narrativa è l'uso del pleonasmo: " $\mathrm{C} i$ incollavano delle immagini di santi a tutte le fessure" (209). " $\mathrm{C} i$ aveva quel po" di dote" (220). "Ora ci hanno messo le cipolle nell'orto" ( 222). "Ci avevano messo sul carro una coperta" (282). "Allora padron 'Ntoni ... si decise a parlare con la Mena di quel che si doveva farsi oramai" (216). "Ognuno che passava per la stradicciuola a quell'ora, udendo il colpettare del telaio di Sant'Agata diceva che l'olio della candela non lo perdeva, comare Maruzza ... . Padron Cipolla confermò che tutti lo sapevan in paese che la Longa aveva saputo educarla la figliuola" (67). Inoltre, come osserva il Caccia (253), è frequente l'uso del pronome maschile al dativo, gli, invece del femminile le.

Per quel che riguarda i modi e i tempi verbali usati dal Verga per rendere ancora una volta l'illusione del dialetto, Giacomo Debenedetti (688), Ettore Caccia (258) e altri hanno rilevato il frequente uso che lo scrittore fa dell'imperfetto per attenersi quanto più fedelmente alla sintassi siciliana e alla narrazione naturalistica intesa come procedimento di accumulazione. In Verga stesso giustifica l'uso insistente di tale tempo verbale durativo in una lettera a Filippo Filippi dell'11 ottobre 1880:

Il mio studio ... d di fare eclissare al possibile lo scrittore, di sostituire la rappresentazione all'osservazione, mettere per quanto si può l'autore fuori del campo d'azione, sicché il disegno acquisti tutto il rilievo e l'effetto da dar completa l'illusione della realtà .... A questo proposito ti dirò che tutti quei passati imperfetti che mi critichi, sono voluti, sono il risultato del mio modo di vedere per rendere completa l'illusione della realtà dell'opera d'arte. (Nencioni 44) 
Riccardo Ambrosini nota il frequente uso del passato remoto in luogo del passato prossimo nell'indicazione di avvenimenti recenti, e la posposizione del verbo come indice di dialettalità (ad es.: "Nulla ho" 69; "Nulla voleva fare lui" 226). Nota anche l'uso delll'imperfetto indicativo in luogo del condizionale composto e del congiuntivo, ad esempio: "Volevano prestati [che fossero prestati loro] gli attrezzi" (Ambrosini 29-31). Inoltre tipico della sintassi siciliana è il complemento oggetto preceduto da "a": ("Sentite a me"), e l'uso di un verbo al posto del sostantivo, come nel periodo seguente: "Ella [la Longa] aveva il viso tutto bagnato; ma non si accorgeva che piangesse", invece "del suo pianto" (206).

Il Nencioni rileva l'uso ostensivo del dimostrativo quello, che dà l'illusione della parlata popolare. ${ }^{9}$ Ancora, come notano il Caccia e l'Ambrosini, sono da rilevare nel campo del lessico vari sicilianismi, per esempio i termini sciara, doglianza, limosina, cataletto, straduzza, malannata, pezzenterie, conocchia, cucco, diavolone, cetriolo, puddara, calcagna, minchione, cristiano, buscare, buscarsi, scialarsi, ecc. (cfr. Caccia 238; Ambrosini 16). Luigi Russo nota come le fortezze della barca siano dette col termine marinaresco "lapazze", termine che pare proprio nato nel dialetto siciliano. I rocchetti del telaio vengono chiamati "cannelli", perché parola più conforme a terminazione verbale siciliana, e l'evangelo è sempre detto "l'evangelio" per rispettare una peculiarità espressiva dialettale delle province orientali della Sicilia; infine l'ateo, il miscredente, viene definito "il protestante" o "l'ebreo" (Russo 358). Per creare l'illusione della realtà di Trezza lo scrittore si serve inoltre di elementi documentari come le nciurii ossia soprannomi che fanno parte della tradizione popolare e che fissano un tratto individuale in una specie d'immobilità perpetua: i Malavoglia, la Zuppidda, la Vespa, la Mangiacarrubbe, Piedipapera, Campana di Legno ecc. Ė anche frequente l'uso dell'appellativo gna' tipico del dialetto: gna' Mena, gna' Grazia, gna' Venera.

Un interessante studio condotto da Gabriella Alfieri analizza le varie trasposizioni italiane di numerosi modi di dire siciliani nei Malavoglia: "si tratta, in genere, di argute similitudini le quali, proprio in vista del loro carattere formulare, si possono considerare vere e proprie frasi proverbiali. Circostan$\mathrm{za}$, certo, non casuale, ma dovuta senz'altro al tentativo, riuscitissimo, del Verga, di far parlare i suoi personaggi come parlano, adoperando espressioni tratte dalla loro stessa vita ed esperienza" (Alfieri, "Innesti" 228). Tra le locuzioni menzionate dall'Alfieri ricordiamo la frase pronunciata dalla pettegola comare Venera quando dice di aver visto 'Ntoni e la Sara di comare Tudda che si parlavano dal muro dell'orto: "con quegli occhi che dovevano mangiarseli i vermi" (58), frase tratta dall'espressione siciliana: L'occhi, si l'avissinu a manciari i vermi. Ancora, la formula siciliana pigghiarasilla ncriminali, viene parafrasata dal Verga con "pigliarsela in criminale", nel senso di prendersela a male per qualsiasi azione ricevuta. ${ }^{10}$

Tra gli altri modi di dire studiati dall'autrice ricordiamo la frase ridiri comu na iaddina tradotta letteralmente con "ridere come una gallina",; an- 
cora, l'espressione "tirare dietro tanto di lingua", parafrasata dal siciliano nesciri tantu di lingua, col valore metaforico di dire liberamente e senza ritegno il fatto suo; ${ }^{12}$ l'esclamazione di 'Ntoni "Carne d'asino, carne da lavoro", tratta da carni di sceccu, carni di travagghiu, o la formula siciliana arristari supra a panza, tradotta letteralmente dal Verga con "rimanere sulla pancia". ${ }^{13}$ Quest'ultimo traslato viene usato nel dialetto siciliano soprattutto per indicare il peso costituito per i genitori da una ragazza che abbia difficoltà a trovare marito.

Numerose altre locuzioni, modi di dire e proverbi sembrano essere trapiantati direttamente dal siciliano, in particolare i proverbi, formule fisse tramandate dagli antichi che rispecchiano una sorta di saggezza extratemporale. ${ }^{14}$ Tra le espressioni e i proverbi che sembrano essere una diretta trasposizione dal dialetto ricordiamo: "Donna di telaio gallina di pollaio, e triglia di gennaio" (56), dal siciliano Fimmina di tilaru, gaddina di puddaru e trigghia di jinnaru (Pitrè 2.82). "Scirocco chiaro e tramontana scura, mettiti in mare senza paura" (63), dal dialetto Sciloccu chiaru e tramuntana scura, mettiti a mari senza paura (Pitrè 3.66). "Amore di soldato poco dura, a tocco di tamburo addio signora" (102), tratto da Amuri di surdatu pocu dura, in tuccari tammuru addiu, signura (Pitrè 1.102). "Matrimoni e vescovadi dal cielo sono destinati" (146), da Matrimonii e viscuvati di lu celu su' calati (Pitrè 2.103). "Buon tempo e mal tempo non dura tutto il tempo" (172), dal dialetto Bon tempu e malu tempu nun dura tuttu un tempu (Pitrè 1.276). "Mare crespo, vento fresco" (172), da Mari crispu, ventu friscu (Pitrè III 40). "Mare bianco, scirocco in campo" (172), dalla fonte dialettale Mari biancu, sciroccu 'ncampu (Pitrè 3.40). "La casa ti abbraccia e ti bacia" (186), da La casa ti strinci e ti vasa (Pitrè 1.220). "Meglio contentarsi che lamentarsi" (203) da Megghiu cuntintarisi, chi lamintarisi (Pitrè 1.305). "Beato quell'uccello che fa il nido al suo paesello" (202), da Biatu chiddu oceddu chi fa lu nidu a lu so' paiseddu (Pitrè 1.216). "Necessità abbassa nobiltà" (218), da La nicissitd sbascia ogni nobiltà (Pitrè 1.200). "Il mondo è tondo, chi nuota e chi va a fondo" (273), da $\mathrm{Na}$ ' rota è stu munnu, cui nata e cui va n' funnu (Pitrè 1.264-65). "Chi fa credenza senza pegno, perde l'amico, la roba e l'ingegno" (81), da Cui fa cridenza senz' aviri pignu, perdi la roba, l'amicu e lu gegnu (Pitrè 2.45). Ricordiamo infine l'espressione "buscarsi il pane", tratta dal siciliano vuscarisi u' pani che ricorre più volte nel romanzo, nel senso di guadagnarsi da vivere.

\section{Italiano e Siciliano: le ambiguità della traduzione nei Malavoglia}

Nonostante gli sforzi dello scrittore siciliano per creare un'atmosfera provinciale-popolare attraverso la costante utilizzazione di sicilianismi, modi e costrutti dialettali, la lingua del Verga resta tuttavia fondamentalmente letteraria e ciò è d'altra parte inevitabile, data la dicotomia esistente tra il livello superiore del narratore e quello primitivo e rozzo della materia rappresentata. Secondo l'Ambrosini:

La quasi totale italianizzazione delle forme siciliane da un lato allontana dalla realtà 
linguistica di fondo, dall'altro rende più sociale che locale il problema che il Verga si era proposto di esporre. Di questi elementi linguistici si coglie piu il livello socioculturale dei popolani di Trezza, che non la precisa identita dialettale. O meglio, il collegamento con quel determinato dialetto di tipo siciliano-occidentale è indicato dalle coordinate CON-TESTUALI delle indicazioni geografiche, dell'onomastica e di usanze e folclore locali, più che da esplicite connotazioni CO-TESTUALI. (38)

Si tratta in definitiva dell'italiano parlato dai siciliani colti della seconda metà dell'ottocento, che attinge sia alle fonti toscane che all'italiano letterario. Non intendo qui dilungarmi a ripetere ciò che è stato già ampliamente trattato in eccellenti studi, ma solo ribadire come la ferma illusione di una lingua dialettale di cui si è parlato non regga di fronte all'utilizzazione di numerose trasposizioni dal dialetto all'italiano le quali, invece di rendere il nucleo semantico dialettale originario, danno luogo a una serie di oscurità semantiche e di ambivalenze interpretative che rivelano la natura fittizia e arbitraria della lingua. Lo studio di Gabriella Alfieri sulla traduzione dei proverbi nei Malavoglia è indicativo della problematica inerente ad ogni tentativo di trasposizione da una lingua all'altra. ${ }^{15}$ L'Alfieri analizza dettagliatamente $\mathrm{i}$ vari mutamenti fonetici, morfologici, lessicali operati dal Verga nella traduzione, i quali, se da un lato frenano l'eccessiva vivacità del dialetto, dall'altro ne limitano la ricca estensione semantica. Com'è possibile, d'altra parte, conciliare l'ingenuità e a volte la crudezza di certe epressioni dialettali con le convenienze stilistiche della lingua italiana? Così, i frequenti tagli di proverbi operati dal Verga, in nome di un'accurata italianizzazione, riducono la ricchezza semantica dell'originale sentenza dialettale: "Senza pilota barca non cammina", (56) estratto da Senza pilota nun camina varca, senza vicinu 'un si cummina furtu (Pitrè 2.284); "A donna alla finestra non far festa" (67), tratto dal siciliano A fimmina 'n finestra 'un fari festa; a fimmina ca ridi, 'un cci aviri fidi (Pitrè 2.61). Tra i mutamenti morfologici menzionati dall'Alfieri, ricordiamo la sostituzione del singolare al plurale nella traduzione dei proverbi. Ad esempio il proverbio siciliano Quannu lu suli si curca 'nsaccatu s'aspettanu li venti di Ponenti (Pitrè 3.55) viene reso con "Quando il sole si corica insaccato si aspetta il vento di Ponente" (64), e Forza di giuvini e cunsigghi di vecchi (Pitrè 2.297), diventa "Forza di giovane e consiglio di vecchio" (218).

Notevole è il trattamento del possessivo, posposto nel sintagma so' casa = "casa sua", con effetti di maggiore incidenza semantica: "Ognuno è padrone in casa sua", o soppresso volendo sottolineare la genericità dello stesso gruppo nominale, per cui il siciliano $\mathrm{Cu}^{\prime}$ è minchiuni si sta a so' casa (Pitrè 3.288) è trasformato in "Chi è minchione se ne sta a casa" (Alfieri, Il motto 128). Si consideri inoltre l'amplificazione del possessivo nel gruppo nominale l'affari soi reso con "gli affari di casa sua". Nell'espressione "Beato chi muore nel proprio letto" (193), lo scrittore trasforma la matrice dialettale so' in "proprio". E ancora nel proverbio 'Ntrdi, 'ntrdi, ognunu cu li soi (Pitrè 1.241), Verga aggiunge il pronome ciascuno: "Ntroi, 'ntroi, ciascuno coi pari suoi" 
(66). Infine, nella formula "Ascolta i vecchi che non la sbagli", lo scrittore sopprime l'originaria particella siciliana ci: Ascuta a li vecchi, cà nun ci la sgarri (Pitrè 2.292).

Per quanto riguarda la flessione verbale, l'Alfieri nota la frequente sostituzione del congiuntivo all'indicativo dialettale. Ad esempio: il siciliano Cui cadi all'acqua è forza chi si vagna (Pitrè 3.70) viene reso con "Chi cade nell'acqua è forza che si bagni" (Verga, Malavoglia 169; Alfieri, Il motto 130-31). Non sfuggirà inoltre a un lettore più accorto l'uso frequente che il Verga fa del futuro nel romanzo, in luogo del presente, corretto nel gergo dialettale. ${ }^{16}$ Parecchie trasformazioni vengono infine attuate nelle combinazioni di pronome e avverbio. Così la corretta inversione dialettale delle particelle si cci viene italianizzata in "ci si", come in "ci si pensa", tradotto dal dialetto si cci pensa, o "ci si appoggia", da si ci appoja (I Malavoglia 132).

Nel campo sintattico Verga riduce a volte due proposizioni indipendenti in due dipendenti implicite, come in "I vicini devono fare come le tegole del tetto, a darsi l'acqua l'uno con l'altro" (93), tratto dal siciliano Li vicini su' comu li catusa, si dunanu acqua l'unu cu l'autru (Pitrè 1.221). Ci sono anche diversi casi di trasformazione di un lessema dialettale con un gruppo sintagmatico italiano come surcera, tradotto con "trappola di topi", catusa con "tegole di tetto", strattu con "conserva di pomidoro" che ha un significato leggermente diverso in italiano, indicando in generale tutti i tipi di salsa, mentre strattu è una salsa particolare fatta con pomodori fatti asciugare al sole. Si noti ancora la sostituzione del siciliano panza col termine letterario "ventre" in questa espressione: "[La Mangiacarrubbe] lo aspettava sulla porta, colle mani sul ventre" (219). Infine, inutile dire che la traduzione rallenta spesso la tensione ritmica e metrica dell'originale proverbio siciliano.

Per quanto riguarda il campo lessicale ciò che ci interessa maggiormente in questa parte del nostro saggio, è sviluppare l'accurata analisi alfieriana, mostrando come, sia che il Verga muti la fonte siciliana, sia che cerchi di tradurre letteralmente certe espressioni e proverbi, lo spettro interpretativo non risulta sempre chiaro e univoco ai lettori siciliani e non-siciliani, dal momento che la traduzione italiana spesso deferisce e disperde il nucleo significativo originale. In altre parole, sottoponendosi al lungo lavorio dell'impresa di rendere in italiano costrutti semantici e sintattici tipici della lingua siciliana, Verga si scontra con la problematica dell'ambiguità e dell'oscillazione interpretativa magistralmente analizzata da Jacques Derrida in una conferenza sulla traduzione, poi raccolta nel volume L'Oreille de l'autre. Derrida dimostra che la traduzione, come tutte le categorie simboliche del pensiero, non riproduce mai un'essenza di significato, bensì tende a rifiettere il sistema di differenze iscritto nell'ordine simbolico. In altre parole, la traduzione, come del resto la lingua in generale, non rende mai una significazione pura e univoca, ma si risolve in un processo di disseminazione, di erranza (différance) di significanti.

Numerosi sono nel testo dei Malavoglia i lessemi e sintagmi che tradotti 
in italiano rischiano di scalzare l'illusione mimetica della lingua popolare, provocando, a una lettura attenta e critica, innumerevoli ambivalenze semantiche. Un esempio si trova nel passo seguente: "Allo Zio Crocifisso gli finiva sempre così, che gli facevano chinare il capo come Peppinino, perché aveva il maledetto vizio di non saper dire di no" (61-62).

L'espressione "chinare il capo come Peppinino" è tratta dall'idioma dialettale Calari a testa comu Peppininu, nel senso originario di piegare di forza, come indicato dal contesto. La trasposizione italiana "chinare il capo" può rendere l'intento della fonte siciliana, come pud allo stesso tempo essere intesa nel senso di abbassare il capo in segno di rispetto. E ancora nella frase "Campana di legno faceva il sordo e dimenava il capo come Tartaglia" (194), tratta dal modo di dire siciliano menzionato sopra, "poco pertinente appare l'accostamento compiuto dal Verga tra il siciliano Peppininu e il toscano Tartaglia, avendo l'uno la caratteristica di non saper reagire alle offese, e quindi di rassegnarsi a tutto, di essere oltremodo condiscendente, e l'altro quella di balbettare" (Alfieri, "Innesti" 242). ${ }^{17}$

Nel proverbio "Carcere, malattie, e necessità, si conosce l'amistà" (103), proveniente dal siciliano Carzari, malittii e nicissitati, scummogghianu lu cori di l'amici (Pitrè 1.91), Verga sostituisce il siciliano scummogghianu (inteso nel senso di scoprono, rivelano) con "si conosce" che contiene varie connotazioni in italiano: potrebbe infatti significare "si distingue", "si riconosce", "si vede", "si rivela". La traduzione provoca di conseguenza un processo di dispersione di significanti. Ancora, nella frase: "I bambini giocavano a nocciuoli nella strada" (111), lo scrittore usa "nocciuoli", invece dell'appropriato femminile nocciuole per essere più conforme al dialetto nuciddi (i frutti del nocciuolo con i quali si suole giocare a Natale). Ma traduce male dal momento che il siciliano non confonde noccioli e nocciuole, detti rispettivamente ossa e nuciddi (Raya 80).

Perfino la traduzione letterale sembra disperdere il senso dell'originale sintagma siciliano, come nel passo seguente: "Tutti si voltarono verso Campana di legno il quale era venuto anche lui, per politica, e stava zitto, in un cantuccio, a veder quello che dicevano, colla bocca aperta e il naso in aria" (91). In generale, l'idioma dialettale pi' pulitica, è usato quando si parla di un'azione che si fa non tanto perché si vuole quanto per adempiere a un dovere sociale. In italiano, invece, l'espressione è ambigua, e non rende totalmente l'originale: oltre a questa implicazione, potrebbe infatti indicare un atteggiamento politico o diplomatico di Campana di legno che vorrebbe venire a un accordo coi Malavoglia per risolvere la faccenda del debito dei lupini. Allo stesso modo una lettura critica rivela un'ambivalenza semantica nel sintagma italianizzato "in capo alla via", contenuto nel seguente passo: "Bastianazzo, che si era sbrigato in fretta dal disarmare la Provvidenza, per andare ad aspettarli in capo alla via, come li vide comparire a quel modo, mogi mogi e con le scarpe in mano, non ebbe animo di aprir bocca, e se ne tornò a casa con loro" (57). "In capo alla via", parafrasato dal siciliano $n$ 
capu a' sta per "sulla via" in dialetto, mentre trasposto in lingua italiana può rivelarsi oscuro, per intendere che Bastianazzo si trova all'inizio della via. Ed in quest'altro passo: "Le comari che sapevano delle chiacchiere fra padron 'Ntoni e compare Cipolla, dicevano che adesso bisognava farle passare la doglia a comare Maruzza, e conchiudere quel matrimonio della Mena" (91). In siciliano l'espressione farici passari a dogghia è intesa nel senso di far passare il dolore, mentre l'italiano appare più ambiguo, essendo il lessema "doglia" usato a volte per indicare dolore fisico o morale, ma principalmente per indicare i dolori del parto.

Una parola parafrasata dal dialetto che viene frequentemente usata dal Verga è il termine "cristiano", inteso originalmente dal dialetto nel senso generico di persona, individuo, "Quel cristiano lo conosceva soltanto di vista e non sapeva altro" (94). "Allora la Longa, come pensava a quell'uomo che moriva di sete in mezzo a tutta quell'acqua, non poteva stare dall'andare ad attaccarsi alla brocca, quasi ce l'avesse avuta dentro di sé quell'arsura, e nel buio spalancava gli occhi, dove ci aveva sempre stampato quel cristiano" (158). "E glielo disse anche in faccia, alla fine, onde levarsi di dosso quella noia, perché quel cristiano stava sempre davanti alla sua porta come un cane" (168). Nella trasposizione italiana il termine si rivela ambivalente, potendo indicare: 1. una persona, un individuo in generale; 2 . uno di religione cristiana. Allo stesso modo, l'idioma siciliano fari u'viagghiu nella traduzione italiana "fare il viaggio", può confondersi col senso letterale del termine, senza trasporre pienamente la significazione dialettale di pellegrinaggio compiuto a piedi nudi per la devozione. ${ }^{18}$

Così il sintagma fari na cummedia, che in siciliano indica una discussione o lite in cui si viene a volte alle mani, dà luogo nella traduzione italiana a varie sfaccettature significative: infatti, oltre a poter implicare il senso metaforico dialettale, può essere inteso in senso letterale (rappresentare una commedia di teatro), o secondo quanto ci riferisce il Petrocchi, fare recitare la commedia si può dire "di chi finga affetti, dolori esageratamente"; o, ancora, secondo il Macaluso-Storaci, può essere riferito a persone, e specialmente a ragazzi che fanno molto chiasso (Alfieri, "Innesti" 260). ${ }^{19}$

Un'ambivalenza semantica emerge ancora nella seguente affermazione di 'Ntoni:

- Io gli rido sul mostaccio a don Michele il brigadiere! Lo so perché ce l'ha con me; ma stavolta può pulirsi la bocca, e farebbe meglio a non sciuparsi le scarpe per passare e ripassare davanti alla Zuppidda, col berretto gallonato, come se ci avesse la corona in capo. (139)

Il senso originale del modo di dire siciliano "pulirsi la bocca", come anche indicato dal contesto, è principalmente quello di "può star fresco, non farà niente!" Tuttavia, la connotazione implicita nel dialetto sembra disperdersi nella traduzione italiana la cui interpretazione vacilla tra il senso figurativo citato e quello letterale (l'azione di pulirsi veramente la bocca). Paul de Man ha 
esaminato questa problematica della continua oscillazione tra significazione retorica (metaforica) e significazione grammaticale, problematizzando i tradizionali preconcetti dell'univocità di senso e delle distinzioni epistemologiche tra grammatica e retorica. ${ }^{20}$

Anche la seguente locuzione vacilla tra significazione metaforica e senso letterale: "Alla spezieria c'erano i soliti sfaccendati, che si dicevano le orazioni, col giomale in mano, o si piantavano le mani sulla faccia, chiacchierando, quasi volessero accapigliarsi" (153-54). Il costrutto "piantare le mani in faccia" è tratto dalla matrice dialettale parrari cu i manu 'nta facci che vuol dire parlare a qualcuno con impeto, assalendolo con rimproveri e parole cattive che lo feriscano (cfr. Alfieri, "Innesti" 257).

Parafrasata in italiano, tuttavia, l'espressione appare un po' oscura e ambivalente, deferendo il nucleo originale: oscilla infatti tra la possibilità di un senso figurativo (che risulta più esplicito in siciliano) e quella di un senso letterale che implicherebbe una vera e propria azione fisica di venire alle mani. Così nell'espressione "un uomo coi baffi" la connotazione metaforica del dialetto di uomo potente, importante, viene offuscata in italiano dal senso letterale: "-Sì, l'avvocato l'ha detto lui che non ci è paura di niente.-Ma cosa ha detto? -insisté Maruzza.-Eh, lui sa dirle le cose; un uomo coi baffi! Benedette quelle venticinque lire!" (114).

L'originale senso figurato della formula dialettale Pirdiri a birritta mmenzu $a$ fudda è quello di non lasciarsi sopraffare, di farsi valere in ogni situazione pericolosa. Tuttavia nella parafrasi italiana l'espressione potrebbe anche alludere a un semplice smarrimento del berretto in mezzo alla folla, come nel seguente contesto: "['Ntoni] lo guardava bene in faccia [Don Michele] ammiccando gli occhi, come deve fare un bravo giovanotto di fegato che è stato soldato, e non si lascia portar via il suo berretto in mezzo alla folla" (139). Si veda ancora il passo seguente: "Padron 'Ntoni ascoltava anche lui, tenendo d'occhio lo scolare della salamoia, e approvava col capo quelli che contavano le storie più belle, e i ragazzi che mostravano di aver giudizio come i grandi nello spiegare gli indovinelli" (200). L'originale fonte dialettale da cui è tratta la frase "spiegare gli indovinelli" è anniminari na 'nniminagghia che vuol dire indovinare. Verga, se da una parte intende eliminare ogni oscurità semantica che limiterebbe l'intelligibilità del modulo, rendendo anniminari con "spiegare", dall'altra crea altre ambivalenze semantiche, in quanto il verbo spiegare potrebbe implicare il significato di indovinare, ma potrebbe allo stesso tempo essere inteso nel senso di illustrare gli indovinelli con esempi, o ancora nel senso di spiegare cosa sono gli indovinelli. Come si vede, l'originario intento della matrice siciliana si disperde in una varietà di sfaccettature semantiche.

Numerosi altri idiomi dialettali, parafrasati in lingua italiana, sembrano perdere la loro originaria connotazione figurata. Tra questi rileviamo l'espressione mettiri 'nto cuntu, nel senso di "considerare" che, tradotta in italiano nella seguente frase si confonde col senso letterale dei termini: "Questa non 
ce l'avevano messa nel conto i Malavoglia!" (208). Anche l'affermazione di padron 'Ntoni, "Sono in sensi", vacilla tra il comune senso metaforico siciliano (siri in sensi=essere padrone delle proprie facoltà), e un oscuro riferimento ai sensi. Ancora, nell'esclamazione di padron 'Ntoni: "Perché vuoi darle questo dolore a tua madre, di vederti fare la riescita di Rocco Spatu?" (233), il termine riescita è chiaramente positivo in italiano, mentre in dialetto presenta a volte una connotazione negativa, come, in questo caso, si riferisce alla brutta fine di Rocco Spatu.

La metonimia siciliana Nun vuliri vidiri a unu nô battisimu, che denota antipatia verso una persona, al punto da non poterne sopportare la vista, si rivela ambigua nella traduzione, dove il senso letterale sembra negare quello retorico. ${ }^{21} \mathrm{E}$ allo stesso modo nell'espressione "Il Signore leva il lume" (da luvari a lumi=togliere il senno) il predominante valore letterale del costrutto trasposto in italiano mette in dubbio la connotazione figurativa. Si deduce dunque da questa analisi che una chiara dicotomia tra livello e collocazione del narratore e realtà sociale rappresentata è inevitabile. Così il fermo rifiuto del Verga di scrivere l'intero romanzo in dialetto siciliano, se da un lato è inteso a rendere intelligibile e accessibile a tutti una materia di per sé grezza quale quella del mondo primitivo dei pescatori di Acitrezza, dall'altro crea innumerevoli ambivalenze semantiche, in quanto la traduzione italiana non sempre rispecchia, ma deferisce e disperde l'originale valore dialettale. La traduzione, in definitiva, riflette l'inevitabile differenza tra significante e significato dando luogo, come conferma l'illustre studioso Henri Meschonnic, a una sorta di décentrement. ${ }^{22}$

Lo scopo principale di questo studio sulla lingua dei Malavoglia è stato quello di mostrare come l'apparente ordine significativo stabilito dalla scienza semiologica e retorica sia continuamente soggetto a un certo dislocamento. Ciononostante non bisogna considerare queste contraddizioni e ambiguità come aspetti negativi dell'arte verghiana, bensì come parte rivelatoria della natura fondamentalmente arbitraria del linguaggio in generale, come tratto problematico di ogni lingua e di ogni tentativo di trasposizione da un idioma all'altro. In altre parole si tratta del dramma della lingua incapace di realizzare gli ideali che lo scrittore si è originariamente proposti. La teoria dell'impersonalità e il principio di mimesis, o imitazione della natura, vengono problematizzati, mettendo ancora una volta in evidenza la dicotomia arte/realtà, e l'apparente coerenza della narrativa realista viene messa in crisi dalla riversibilità e dalla pluralità dei codici interpretativi. Così, se chi legge I Malavoglia si illude per un momento di avvertire in quelle cantilene dialettali, "tutto un sentire pregnante o dell'amarezza del vinto o dell'ardore avido del barbaro" (Russo 326), a una lettura più attenta e critica l'illusione del dialetto è soggetta a demistificazione, accentuando l'impossibilità della lingua di cogliere l'intima essenza della realtà e l'impossibilità del letterato di penetrare pienamente nel mondo popolare siciliano mostrando un atteggiamento nei confronti di quel mondo che non sia di tipo paternalistico o pietistico. 
L'opera letteraria non è più quindi valutata come una struttura delimitata che tende a un'oggettività scientifica, bensì come un'entità soggetta a contraddizioni e ambiguita e aperta a diverse possibilita interpretative. Il processo di significazione e di traduzione si risolve in un continuo fluttuare di significanti e di disseminazione di sensi che problematizzano la funzione stessa della lingua e non sono mai in grado di rappresentare o trasporre un nucleo originario intatto, bensì evidenziano il continuo stato di desiderio di un'essenza significativa da parte dello scrittore e del lettore, come ben ci illustra Jacques Derrida:

Le désir du noyau intact, c'est le désir, c'est-à-dire que c'est irréductible; il y a un rapport au noyau intact qui est pré-historique, pré-originaire, qui est ce à partir de quoi un désir quelconque peut se constituer. Donc le désir ou le phantasme du noyau intact est irréductible, mais il n'y a pas de noyau intact. . . . Ce désir du noyau intact, qui meut toute espèce de désir, toute espèce de langue, toute espèce d'appel, toute espèce d'adresse, et cela est la nécessité dure, c'est une nécessité terrible. (153)

\section{Louisiana State University}

\section{NOTE}

1 Per Masiello, ad esempio, "paradossalmente, ad ur massimo di oggettività rappresentativa, mimetica, s'accompagna un massimo di trasfigurazione formale, un massimo di distacco ideale". (109).

2 Si veda Verga, "L'Amante di Gramigna", in Vita dei campi: "Ogni sua parte sarà così completa che il processo della creazione rimarrà un mistero ...., il suo modo e la sua ragione di essere cosł̀ necessarie che la mano dell'artista rimarrà assolutamente invisibile . . . allora avrà l'impronta dell'avvenimento reale; l'opera d'arte sembrerà essersi fatta da sé" (202).

3 Per un'ampia e precisa enunciazione delle teorie naturaliste francesi si veda Zola: "Toute l'opération consiste à prendre les faits dans la nature, puis à étudier le mécanisme des faits en agissant sur eux par les modifications des circonstances et des milieux, sans jamais s'écarter des lois de la nature" (8).

4 Secondo il Masiello, esiste una dicotomia fondamentale tra livello del narratore e realta sociale rappresentata: il piano colto del narratore borghese e il piano rozzo, illetterato della gente di Acitrezza. La difficoltà principale del Verga era quella di trovare "soluzioni linguistiche e stilistiche capaci di colmare il dislivello, di mediare i due piani riducendo al minimo gli scarti. Impresa certo non agevole, perseguita attraverso una ricerca, una sperimentazione assidua e costante" (90).

5 Come osserva il Nencioni, "le pagine dei Malavoglia sono generalmente costruite con lunghi periodi, in cui proposizioni e immagini s'infilano una dopo l'altra con nessi deboli e vaghi, con soste inattese e allungamenti imprevisti sino a clausole che non hanno più relazioni col principio" (30).

6 Per il Bally tale tecnica narrativa è di origine dotta, per lo Spitzer è di origine popolare. Ecco alcuni esempi di Erlebte Rede nei Malavoglia: "Ma se volevano truffargli la sua roba, col pretesto che Bastianazzo s'era annegato, la truffavano a Cristo, com'è vero Dio! Ché quello era un credito sacrosanto come l'ostia consacrata, e quelle cinquecento lire ei l'appendeva ai piedi di Gesù crocifisso; ma santo diavolone! Padron 'Ntoni sarebbe andato in galera! La legge c'era anche a Trezza!" (85). "Donna Rosolina intanto raccontava a Don Silvestro le grosse faccende che ci aveva per le mani: dieci canne di ordito sul telaio, i legumi da 
seccare per l'inverno, la conserva di pomidoro da fare, che lei ci aveva un segreto tutto suo per avere la conserva di pomidoro fresca tutto l'inverno" (88). "Sua moglie gli piantava le unghie in faccia, e gli gridava che voleva avere le chiavi lei, e non voleva star sempre a desiderare un pezzo di pane e un fazzoletto nuovo peggio di prima; perché se avesse saputo quel che doveva venire dal matrimonio, con quel bel marito che le era toccato, si sarebbe tenuta la chiusa e la medaglia di Figlia di Maria!" (237).

7 Anche Nencioni nota nei Malavoglia uno scarso sviluppo della subordinazione e un accrescimento del periodo per coordinazione illogica (28).

8 A proposito del periodare verghiano nei Malavoglia, il Caccia nota come "la forma più viva dello stile verghiano si ha quando la sintassi spezzettata, frammentaria, a continue riprese, vuole esprimere il modo di una narrazione popolare in cui tutto è impressione di vita, immediata, coloristica, ma anche disordinata, impulsiva, senza alcun controllo razionale" (261).

9 Il Nencioni rileva espressioni come: "Quel furbaccio di Campana di legno", "quella civetta della Sant'Agata", "quell'elefante di Mastro Turi", "quella brutta Vespaccia" (49).

10 Cfr. I Malavoglia: "Compare Piedipapera si divertiva a sparlare di questo e di quello, come capitava; ma così di cuore, e senza malizia, che non c'era verso di pigliarsela in criminale" (64).

11 I Malavoglia: "Per questo nel paese volevano fargli la festa allo speziale; ma lui si metteva a ridere come una gallina, preciso come faceva don Silvestro" (211).

12 I Malavoglia: "E brontolava ancora per la strada, mentre Piedipapera chiudeva l'uscio, tirandole dietro tanto di lingua" (137).

13 I Malavoglia: "Carne d'asino!-borbottava;-ecco cosa siamo!-Came da lavoro!" (203). "Le ragazze si maritano così, se no vi restano sulla pancia come le casseruole vecchie" (111).

14 Come è ben saputo, il Verga si serve dei volumi del Pitrè. Per la traduzione dei suoi proverbi si avvale di alcuni dizionari, in particolare del Novo vocabolario siciliano-italiano del Traina e del Vocabolario italiano della lingua parlata del Rigutini.

15 L'Alfieri nota: "Nell'atto del trasferimento dal dialetto alla lingua, l'andata di modifiche investe il proverbio nella sua globalita: le formule dell'intero corpus risultano mutate nell'aspetto complessivo rispetto alla veste originaria, poiché i singoli elementi (monemi, lessemi o sintag. mi) risultano interessati alle necessarie commutazioni fonetiche, lessicali o morfosintattiche, secondo le corrispondenze più o meno immediate ed essenziali fra siciliano e italiano" (122).

16 L'insistente uso del futuro emerge in particolare nel seguente discorso di Padron 'Ntoni: "Non fate tante spese quando non ci sard piu. Il Signore lo sa che non possiamo spendere e si contenterd del Rosario che mi diranno Maruzza e la Mena. Tu, Mena, ... ti terrai sotto le ali tua sorella, come fa la chioccia coi suoi pulcini. Finché vi aiuterete l'un l'altro, i guai vi parranno meno gravi. Ora 'Ntoni è grande e sard in grado di aiutarvi anche lui" (180).

17 L'Alfieri sostiene che la differenza tra i due personaggi è ben rivelata dal Camazzi in un recente commento ai Malavoglia: "Peppinino: maschera siciliana. Nofriu e Peppi-e-Ninu erano tipici del teatro dei pupi"; "Tartaglia: maschera della Commedia dell'Arte. Rappresenta il tipo del vecchio notabile (giudice, dottore, ecc.) sciocco, presuntuoso e balbuziente" (122).

$18 \mathrm{Si}$ veda il passo dei Malavoglia: "Diceva che ei [lo zio Crocifisso] faceva il viaggio alla casa del nespolo come quelli che hanno fatto il voto alla Madonna dell'Ognina" (96).

19 Si veda il seguente passo dei Malavoglia dove si pud riscontrare l'espressione: "La mamma dice che le scarpe inverniciate son fatte per mangiarci la dote e ogni cosa; e qualche bel giorno vuole uscire fuori sulla strada, colla rocca in mano, a fare una commedia con quel Don Silvestro, se non mi lascia in pace" (118).

20 Servendosi di vari esempi, De Man viene alla conclusione che "A perfectly clear syntactical paradigm engenders a sentence that has at least two meanings, of which the one asserts and the other denies its own illocutionary mode" (10).

21 Cfr. I Malavoglia: "['Ntoni] poi si levava di là frettoloso, e se ne andava all'osteria a smaltire 
l'uggia, e se incontrava quelli del berretto gallonato, faceva il giro più lungo per non vederli neanche nel battesimo" (244).

22 Cfr. Meschonic 313-14: "La traduction n'est plus définie comme transport du texte de départ dans la littérature d'arrivée ou inversement transport du lecteur d'arrivée dans le texte de départ, ... mais comme travail dans la langue, décentrement, rapport interpoétique entre valeur et signification, structuration d'un sujet, et non plus sens".

\section{OPERE CITATE}

Alfieri, Gabriella. "Innesti fraseologici siciliani nei Malovoglia". Bollettino del Centro di studi filologici e linguistici siciliani 14 (1980): 221-95.

Il motto degli antichi. Proverbi e contesto nei Malavoglia. Catania: Fondazione Verga, 1983.

Ambrosini, Riccardo. "Proposte di critica linguistica. La dialettalità nel Verga". Linguistica e letteratura 2 (1977): 7-48.

Caccia, Ettore. "Il linguaggio dei Malavoglia". Tecniche e valori dal Manzoni al Verga. Firenze: Olschki Editore, 1969. 229-64.

Cecchetti, Giovanni. "Verga and Verismo: the Search for Style and Language". Petrarch to Pirandello: Studies in Italian Literature in Honour of Beatrice Corrigan. Ed. Julius A. Molinaro. Toronto \& Buffalo: U of Toronto P, 1973. 169-85.

De Man, Paul. "Semiology and Rhetoric". Allegories of Reading. Figural Language in Rousseau, Nietzche, Rilke, and Proust. New Haven and London: Yale UP, 1979.

Debenedetti, Giacomo. Il romanzo del novecento. Quaderni inediti. Milano: Garzanti, 1971.

Derrida, Jacques. L'oreille de l'autre. Montreal: VLB Editeur, 1982.

Devoto, Giacomo. "I piani del racconto in due capitoli dei Malavoglia". Bollettino del Centro di studi filologici e linguistici siciliani 2 (1954): 5-13.

Profilo di storia linguistica italiana. IV ed. Firenze: La Nuova Italia, 1971.

Giachery, Enrico. "Considerazioni sul periodo lungo verghiano". Verga e D'Annunzio. Milano: Silva, 1968. 21-115.

Masiello, Vitilio. "La lingua del Verga tra mimesi dialettale e realismo critico". Il caso Verga.

Ed. Alberto Asor Rosa. II ed. Milano: Palumbo Editore, 1972. 89-117.

Meschonic, Henry. Pour la poétique ll. Paris: Gallimard, 1973.

Nencioni, Giovanni. La lingua dei Malavoglia e altri scritti di prosa, poesia e memoria. Napoli: Morano, 1988.

Pitrè, Giuseppe. Proverbi siciliani raffrontati con quelli di altre regioni d'Italia. Palermo: Il Vespro, 1880.

Raya, Gino. La lingua del Verga. Firenze: Le Monnier, 1969.

Russo, Luigi. Giovanni Verga. Bari: Laterza, 1959.

Sapegno, Natalino. Appunti per un saggio sul Verga. Ritratto di Manzoni. Bari: Laterza, 1986. Spitzer, Leo. "L'originalita della narrazione nei Malavoglia". Studi italiani. Milano: Vita e

Pensiero, 1976. 292-316.

Verga, Giovanni. Lettere al Capuana. Ed. G. Raya. Firenze: Le Monnier, 1975.

I Malavoglia. Ed. Corrado Simioni. Milano: Mondadori, 1971.

I Malavoglia. Introd. e note di G. Camazzi. Milano: 1978.

Vita dei Campi. Milano: Mondadori, 1979.

Zola, Emile. Le roman expérimental. Paris: E. Fasquelle, 1923. 